\title{
Estimation of intercity freight origin-destination matrix using simulated annealing algorithm
}

\author{
Mohsen Sadeghi ${ }^{\mathrm{a}}$ and Gholamali Shafabakhsh ${ }^{\mathrm{b}^{*}}$
}

${ }^{a}$ Ph.D. Candidate, Department of Civil Engineering, Semnan University, Semnan, Iran

${ }^{b}$ Professor, Department of Civil Engineering, Semnan University, Semnan, Iran

\begin{tabular}{l}
\hline C H R O N I C L E \\
\hline Article history: \\
Received December 2, 2016 \\
Received in revised format \\
December 10, 2016 \\
Accepted June 12017 \\
Available online \\
June 12017 \\
\hline Keywords: \\
Estimation of freight O-D \\
matrix \\
Logistic \\
Intercity transportation, \\
assignment \\
Traffic count \\
Simulated annealing \\
algorithm \\
Commercial vehicles \\
\hline
\end{tabular}

\section{A B S T R A C T}

The information of trip demand as the freight origin-destination (O-D) matrices plays a significant role in intercity transportation engineering and planning. Access to this matrix from conventional methods needs to spend time, money and human resources. During the recent years, numerous studies have been conducted in order to obtain the freight O-D matrix using freight vehicle count information in network links. Furthermore, the estimation of O-D matrix and its communicating with freight vehicles can greatly help planners improve logistic, safety and the maintenance issues. This paper presents a method based on the minimization of the deviations between the observed and estimated values for finding the freight O-D matrix. To solve the model, traffic count data of trucks in the network links as well as other related information resources are used. The model is solved for a case study in Iran using metaheuristic Simulated Annealing Algorithm.

\section{Introduction}

During the last two decades, the estimation of O-D matrix or trip table based on the existing information has increasingly been the focus of attention in the field of researches. O-D matrix is a fundamental input for transportation planning. Elements of this matrix represent the number of trips between different zones throughout a city, a province or a country during a given time period. The high costs of production of such matrices from direct methods and survey have led many researchers to use other methods. Assigning an O-D matrix to transport network will lead to traffic flow in the network links, while O-D matrix can be obtained from traffic count in network links and actually a reverse action is performed. Numerous approaches have been applied for estimation of OD matrix in the recent decades (Wang et al., 2016) and most of them use the link traffic count, directly. Conventional methods include entropy maximization method (Van Zuylen \& Willumsen, 1980), maximum likelihood estimation (Spiess, 1987), estimation of O-D matrix (Cascetta \& Nguyen, 1988), Generalized Least Squares (GLS) (Bell, 1991) and Bayesian inference estimation techniques (Maher, 1983).

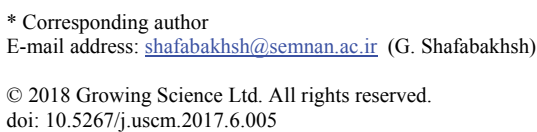


Given that most researches in this field have been based on urban and passenger journeys, a method is presented in this study for freight O-D matrix for a country. The purpose of this study is to estimate the freight O-D matrix for different periods (monthly, multi-month or annually) using a model, where inputs include freight commercial vehicle count in network links and primary information obtained from field surveys at different periods in Iran. This model is solved using Simulated Annealing Algorithm and it is tried to use different sources for estimating the freight O-D matrix better.

After a review of previous studies, the objectives of this research become also clear and the O-D matrix estimation model is offered. This model which is based on minimizing the deviations between the observed and estimated values, have been employed in many O-D matrix estimation studies. It has been also used in this paper with applying changes in the model and particularly the conversion of its parameters to the parameters associated with the freight transport and making it intercity. Cohen (1995) applied two series of data to develop the freight transport model, which were primary and secondary data. The primary data includes network traffic counts and the secondary data includes periodic surveys.

For this reason, in this paper, the road transport network information of Iran, location and data of traffic counters are considered as the primary data. In addition, some coefficients such as empty trucks between each pair of O-D and the average load of each 2-axle, 3-axle and more truck for each pair O$\mathrm{D}$ are considered as the secondary. These data are obtained from the surveys utilized to offer a desirable freight O-D matrix estimation. The final section also provides a summary of research efforts and recommendations for future researches.

\section{Literature Review}

Although many efforts in O-D matrix estimation models have focused on passenger vehicles, since the early 1990s, the O-D matrix estimation of trucks has attracted much attention. The freight O-D matrix estimation methods are associated with freight transport vehicles and trucks are almost identical in structure with passenger estimation methods. Numerous studies have been carried out in conjunction to estimate origin-destination matrix of freight or to determine the table of movements of trucks between origin-destination, too. Since the estimate of OD matrix of freight is completely different from origin-destination matrix of the passenger, in most studies in addition to the use of the data associated with traffic volume counts, another database is also used (e.g. Shabani \& Figliozzi, 2012). The database information as well as the related surveys should be used to determine some points such as recognition of empty trucks behavior and the average load occupation factor of each truck type. In these models, the aim is to estimate the O-D matrix which produces flows having most compatible with the actual and observed data. Tamin and Willumsen (1989) used a three-step model to estimate the demand for freight flow based on the implementation of gravity and least squares models.

Crainic et al. (2001) used a bi-level program to adjust target freight demand matrix. Its high level was to minimize the difference between the number of observed and estimated trucks and the low level of the traffic estimation model was considered as the system optimize method. Such data includes any combination type of traffic count of the selected links, O-D matrices of other time periods, trip-start and trip-end depending on each zone, and counting of cutting lines (List et al., 2002). List and Tranquist (1994) conducted the first research using such methods for estimating the O-D matrix of trucks movement. They offered a method for estimating truck trip matrices in multiple classes based on the observations of traffic volume and some other information. Data sets of widely varying character are combined in an efficient and effective manner so that each piece of information plays a role in developing the estimated flows. List and Turnquist's model (1994) dealt with the estimation of trip matrices as a linear minimization problem in large-scale, and the goal was to minimize all deviations of estimated values from the observed values. The model provides the greatest possible use from the available information. According to List and Turnquist's research (1994), Réos et al. (2002) assessed the importance of different information categories in the freight O-D matrix estimation. According to 
this study, it was concluded that, traffic count data of the link could be regarded as the most useful information section and since then the statistics of the origin trip production and destination trip absorption are placed as aggregated. List et al. (2002) suggested a model for estimating truck flows for the New York City area. Although the model was formed based on the work of List and Turnquist (1994), it had some progresses over the previous model by adding new types of observations to the previous sets of observations. Zhang et al. (2004) obtained intermodal flow including road, rail and inland river for the state of Mississippi using CFS data and TRANSEARCH data. Al-Battaineh and Kaysi (2005) also used genetic algorithm for an optimization problem where the objective function was minimization of the least squares between the observations and the estimated data to estimate freight OD matrix. Jansuwan et al. (2016) offered a two-step approach to estimate trip table of trucks. In fact, they used freight flow and the data related to counting the volume of trucks. In the first step, the freightbased origin-destination trip table of trucks was estimated through freight flow database. Then, in the second step, the trip table of trucks was rectified by using path flow estimator to obtain the best match by counting the volume of trucks. They made the estimate about UTAH State and made use of FAF and USTM databases. In the literature of their research they also pointed out that, basically, estimate models, freight origin - destination matrix were either trip based or freight based.

Although more data are used nowadays to estimate freight origin-destination matrix particularly in developed countries such as GPS data in trucks. GPS data show the position of trucks in different areas; however, they are not able to generate load occupation factor, or to determine whether trucks are empty or not and/or some other points. Ma et al. (2011), Pinjari et al. (2013) and Flaskou et al. (2015) were among the researchers who did some studies to produce load flow. However, due to the lack of such data in Iran and the lack of GPS in trucks it is not possible to apply such models.

This article is formed based on modeling techniques using traffic count of trucks and commercial vehicles to be able to further improve the freight transport modeling in a large area. Approaching the estimated O-D matrix to the observed matrix in a large scale is considered as part of the objective function of a minimization problem in this study. The data associated with the freight transport in Iran was used as much as possible, and finally the model was employed for the Iran's transport network.

\section{Research Methodology}

Several methods have been presented for the O-D matrix estimation of traffic volume of network links; examples of these methods include maximum likelihood method (Spiess, 1987), generalized least squares (Cascetta, 1984), and bays inferential estimation techniques (Maher, 1983). All these methods have the same goal that is to minimize the error between observed values and estimated values from the model.

The main difference between the estimation of freight and passenger matrices in these models is that in the estimation of passenger origin-destination matrix there is actually no empty vehicle and the driver is considered as a passenger. However, in the estimation of freight origin-destination matrix, first, we counting the volume of different kinds of trucks is important; second, load occupation factor for each kind of truck (different kinds of trucks that are counted) and finally the ratio of empty trucks to total passing trucks of any kind are particularly important.

For example, in California in the United States and in the census of traffic, traffic data particularly for trucks is provided based on the number of their axels including trucks with 2, 3, 4, and 5 axels and more, while there is not such data for passenger vehicles. Meanwhile, in Iran the same data is recorded just for trucks with 2, 3, and more axels. Therefore, the sum of these points shows the difference between solving the problem for estimating passenger and freight truck matrices.

In other presented papers in this field, the observed, estimated urban travel O-D matrix and the traffic count of commercial vehicles carrying freight were applied. In Iran, traffic volumes in the points that traffic count is carried out in a mechanized way and with traffic identifiers are completely segregated 
and, in the category of freight vehicles, vehicles are divided into two categories: 2-axle trucks, 3- axle trucks and more-axle trucks. In addition, factors of empty trucks and average load for types of trucks are obtained between each pair of O-D in two time intervals with field surveys. Other parameters where their descriptions are presented in the following are obtained from computational methods and the traffic assignment. The share of traffic assignment of each pair O-D from each link is among them. Therefore, in this study, it has been tried to use all the available information. Moreover, all or nothing assignment model has been utilized for traffic assignment. The reason of this matter is the lack of multiple routes between origins and destinations. Unlike congested and crowded urban routes, in urban road network, users prefer to use the shortest route due to absence of congestion. In this article, the Simulated Annealing Algorithm has been used as a search method for determining the absolute optimum response in minimization problem. Finally, this problem can be formulated as follows,

$$
\min \gamma_{1} \sum_{i j}\left(T_{i j}-\hat{T}_{i j}\right)^{2}+\gamma_{2} \sum_{a}\left(v_{a 2}-\hat{v}_{a 2}\right)^{2}+\gamma_{2} \sum_{a}\left(v_{a 3}-\hat{v}_{a 3}\right)^{2}
$$

subject to

$$
\begin{array}{lr}
\hat{R}=\operatorname{Assign}\left(\hat{T}_{i j}\right) & \\
\hat{R}_{a i j}=p_{a i j} \hat{R}_{a} & \forall a, i j \\
\hat{f}_{a k i j}=\alpha_{a k i j} \hat{R}_{a i j} & \forall a, i j \quad k=2,3 \\
\hat{v}_{a k i j}=\delta_{a k i j} \hat{f}_{a k i j} & \forall a, i j \quad k=2,3 \\
\hat{v}_{a k}=\sum_{i j} \hat{v}_{a k i j}+\hat{e}_{a k i j} & \forall a, i j \quad k=2,3
\end{array}
$$

where, $\gamma_{1} \& \gamma_{2}$ reflects the importance of two parts of the objective function consisted of freight O-D matrix and traffic counts. $T_{i j}$ is the initial freight O-D matrix, $\widehat{T}_{i j}$ is the estimated freight matrix, $\hat{R}$ is the freight vector obtained from matrix assignment $\widehat{T}_{i j}$ to network links. $\hat{R}_{a}$ is the freight volume in the link $a . p_{a i j}$ is the share of O-D pair $i j$ from the link of passing flow volume from link $a . \hat{R}_{a i j}$ is the freight value that are flowed in between O-D pair $i j$ in the link $a . v_{a 2}$ is the number of 2-axle trucks counted in the link $a . v_{a 3}$ is the number of 3 -axle and more trucks counted in the link $a . \hat{v}_{a 2}$ is the number of computational 2-axle trucks (via matrix assignment $\hat{T}_{i j}$ ) and $\hat{v}_{a 3}$ is the number of computational 3-axle and more trucks (via matrix assignment $\widehat{T}_{i j}$ ) in the link $a$. Assign $\left(\widehat{T}_{i j}\right)$ is the function of assigning freight to the network. $K$ index is related to the type of truck (2-axle or 3-axle and more). $\hat{f}_{a k i j}$ is the freight carried by the truck type $k$ in the link $a$ between the O-D pair $i j$. The factor $\alpha_{a k i j}$ divides the value of current freight in the link between two types of vehicles.

The coefficient is obtained from the database of weigh in motion systems as well as traditional weighing for 212 links. To obtain the coefficient for other links of the network and for each origin-destination pair, the mean value of arcs is used for which statistics have been conducted. Thus, the active paths of each origin-destination pair and its links have been identified and the mean coefficient is used for the whole links for which no data is available. $\hat{v}_{a k i j}$ is the number of full trucks of type $k$ in link $a$ between origin-destination pair $i j . \delta_{a k i j}$ is the coefficient of converting load to truck for the trucks of type $k$, between origin-destination pair $i j$. This coefficient is also obtained like the previous one and from the same database; moreover, the same data have been used for the links the data of which was available; and for the other links it has been calculated in the same way as the previous method. Finally, $\hat{e}_{a k i j}$ is the number of empty trucks of type $k$ in link $k$ between origin-destination pair $i j$ which is obtained from the same database and is a ratio of full trucks of type $k$ in each link. The coefficient is directly obtained for the links with weigh in motion systems and for the other links it can be calculated through the same way as the abovementioned one.

Fig. 1 illustrates the flowchart of the model solution. 


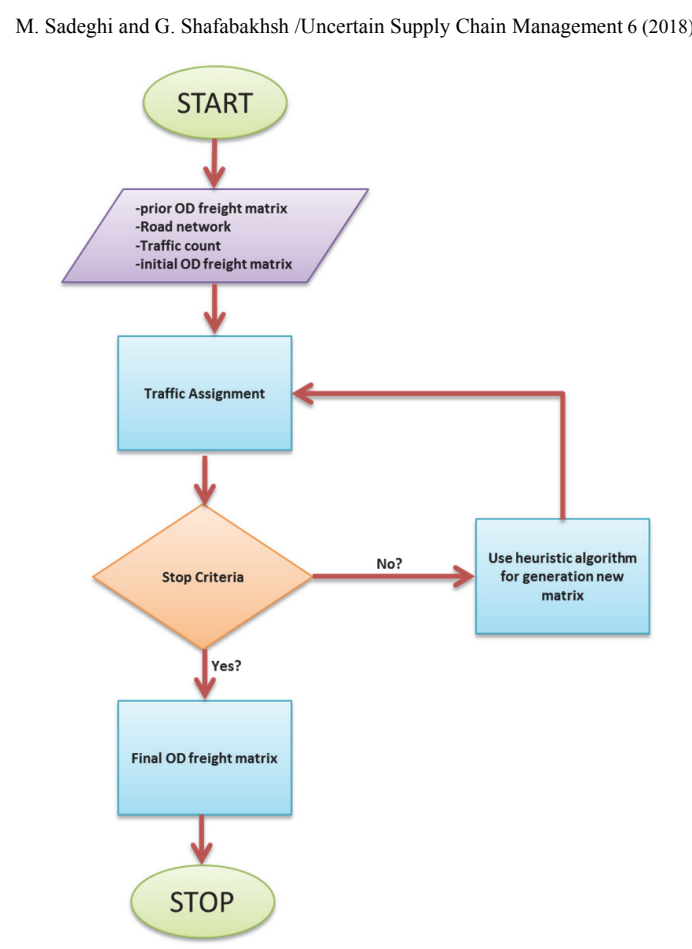

Fig. 1. Flowchart of the model solution

\section{Solution Method}

The aim of this study is to improve freight transport modeling across a country through a method based on traffic count of commercial vehicles and trucks in network links using simulated annealing algorithm as the optimization engine. The simulated annealing algorithm is a simple, effective and meta-heuristic optimization algorithm for solving optimization problems. This method is based on Monte Carlo optimization method which presented by Metropolis et al. (1953). In, Kirkpatrick et al. (1983, 1985) used the simulated annealing algorithm for solving a complicated combinatorial optimization problem. Since then, the simulated annealing method has been employed for solving various problems including many independent variables. Among the problems related to transportation research which have been solved by simulated annealing are traveling salesman problem (Cerny, 1985), vehicle routing problem (Woch \& Lebkowski, 2009), transit scheduling (Poorjafari \& Holyoak, 2014) and network design (Jayaraman \& Ross, 2003).

Gradual annealing technique is used by metallurgists to achieve a state in which solid substance is well arranged and its energy is minimized. This technique involves placing the material at high temperatures and then a gradual temperature reduction. Unlike local optimization methods that can only find a minimum value relative to the initial guessed response, the simulated annealing method can find the absolute minimum value. The main concept of the simulated annealing method is originated from the physical process of refrigeration of molten metals. In the refrigeration process, a molten metal is gradually cooled at very high temperatures. Metal atoms are placed randomly at high-temperature and thus can easily be moved towards one another. By gradually decreasing temperature, the movement of atoms is limited so that atoms start to be sorted and form crystal. The energy level of formed crystal depends on the cooling speed of metal. If temperature reduction is done quickly, crystal structure may not be formed and instead a non-crystal structure is formed with high energy level. Hence, to achieve the lowest energy level, the cooling process must be done slowly.

The simulated annealing method finds the absolute minimum value of an objective function using the simulation of the process mentioned above. The objective function is equivalent to the energy level which should be minimized using a series of optimizer changes. In this method, the cooling process is 
simulated based on the Boltzmann distribution function by controlling a pseudo temperature parameter. Slow reduction of temperature is equivalent to the acceptance of non-optimizer temperature changes with a certain probability that is decreased by reduction of the objective function value. The simulated annealing method uses Boltzmann distribution as shown in Eq. (7), where, $E$ and $T$ represent the energy and system temperature, respectively. Also, $k_{b}$ represents Boltzmann's constant. This distribution asserts that when a system is in thermal equilibrium at temperature $T$, it has an energy distribution that is distributed among all various energy states. There is always the possibility that even in a lowtemperature the energy state of system is high. Thus, this chance exists that the system comes out of the local minimum energy value and converges to an absolute value.

$$
\mathrm{P}(E)=\mathrm{e}^{\left(-\frac{E}{k_{b} T}\right)}
$$

Solution Algorithm is shown in Fig. 2.

Step 1: Select an initial response $i, \mathrm{k}=1$ (counter parameter), an initial temperature and $L$ parameter.

Step 2: Repeat steps 3 to $6 \mathrm{~L}$ times.

Step 3: Select the response $j$ in the neighborhood $i$.

Step 4: Calculate $\Delta C_{i j}=\frac{C(j)-C(i)}{C(i)}$.

Step 5: If $\Delta C_{i j} \leq 0$ or $\exp \left(\frac{-\Delta C_{i j}}{T_{k}}\right)>\operatorname{Rand}[0,1)$ insert $i=j$ and go to the step 6 .

Step 6: $k=k+1$

Step 7: Reduce the temperature $T_{k+1}=f\left(T_{k}\right)$ and go to the step 2 .

$f\left(T_{k}\right)=r^{k+1} * T_{k}$

Fig. 2. Simulated Annealing Method Algorithm

Let $T_{i j}$ be the initial freight O-D matrix for one given year, set of traffic volumes in network links for 2-axle, 3-axle and more trucks that are denoted by $v_{a 2}$ and $v_{a 3}$ as well, have been extracted for the same year for all points with traffic counts. Now, it is needed to follow an initial response for the matrix $\hat{T}_{i j}$. For this reason, the elements of matrix $T_{i j}$ are changed randomly to create a new matrix such as $T_{i j}^{0}$. The way of the work is that each element of the matrix is randomly changed so that its corresponding element in the matrix $T_{i j}$ has a maximum percent $\beta$ increase or $\beta$ percent decrease, i.e.

$$
\left(1-\frac{\beta}{100}\right) * T_{i j} \leq T_{i j}^{0} \leq\left(1+\frac{\beta}{100}\right) * T_{i j} .
$$

Now a corrupted matrix is obtained where each element of it is randomly changed up to $\beta$ percent from the top and bottom relative to the matrix. This matrix will be used as the initial response. To change the response and create a new response, by indexing matrix elements from 1 to the square of the zone number, 4 elements are selected among elements that have reduced and 4 elements are selected among elements that have increased randomly. Then, these 8 elements will be corrected in accordance with the following equations:

$\theta_{i j}=0.1 * T_{i j}^{0}$

$T_{i j}^{f}=T_{i j}^{0}-\theta_{i j} \quad$ For elements that have increased relative to the initial matrix element

$T_{i j}^{f}=T_{i j}^{0}+\theta_{i j}$

For elements that have decreased relative to the initial matrix element.

Again the sum of rows and columns of the matrix that has not been changed must be returned to the initial value until no change occurs in the sum of rows and columns of the matrix relative to the initial matrix. The initial and final temperature value must be determined according to the structure of the problem, so that the probability of accepting bad response should be high at the beginning of algorithm 
iterations and it must be very low at the end. Therefore, the value of the objective function was calculated for the first time and then the temperature was regarded to have the above conditions. As a result, the initial temperature was selected to be 0.1 and the value of $L$ was 100 . For the temperature reduction, the exponential function described in algorithm with $r=0.95$ and a decrease of 1,000 times in temperature are used.

\begin{tabular}{|l|l|l|l|l|l|l|l|}
\hline & 1 & $\ldots$ & $\ldots$ & $\ldots$ & $\ldots$ & $\ldots$ & $\begin{array}{l}\text { Number of last } \\
\text { zone }\end{array}$ \\
\hline 1 & & & & & & & \\
\hline$\ldots$ & & $+\theta_{i j}$ & $-\theta_{i j}$ & & & $-\theta_{i j}$ & \\
\hline$\ldots$ & & & & & & & \\
\hline$\ldots$ & $-\theta_{i j}$ & & & $+\theta_{i j}$ & & & \\
\hline$\ldots$ & & & & & $+\theta_{i j}$ & & $-\theta_{i j}$ \\
\hline $\begin{array}{l}\text { Number of last } \\
\text { zone }\end{array}$ & & $+\theta_{i j}$ & & & & & \\
\hline
\end{tabular}

Fig. 3. Building of solution $\mathrm{T}_{\mathrm{ij}}^{\mathrm{f}}$ in the neighbourhood of a given solution $\mathrm{T}_{\mathrm{ij}}^{0}$

\section{A Case Study in Iran}

The proposed model has been implemented for Iran's road network with a length of $34600 \mathrm{Km}$ and also 56 traffic zones. Due to conducting traffic count for 1165 sections in the mentioned network and specified number of 2-axle, 3-axle and more trucks, on each of these points, these data have been employed as input data. In this paper all of the traffic count data in Iran have been used, although the number of links could be propounded as a sub problem. Khan and Anderson (2016) reviewed the limitation of the number of traffic count links in the estimated O-D matrix accuracy. Moreover, the freight O-D matrix that is obtained from field surveys of the Ministry of Roads and Urban Development in 2011 has been used.

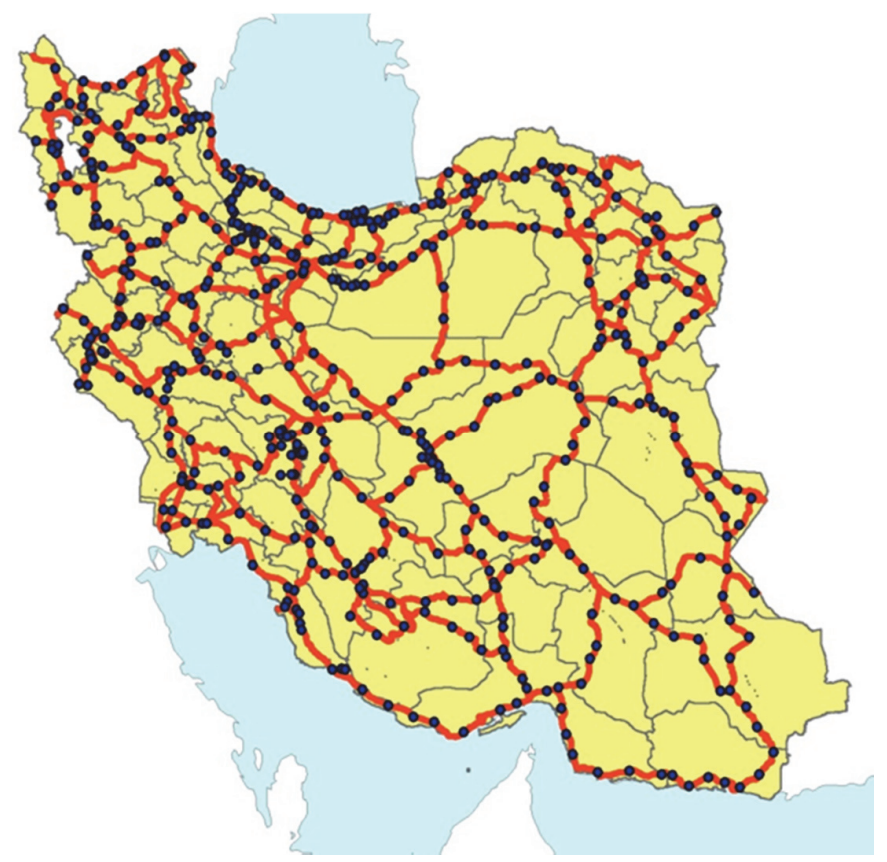

Fig. 4. Iran arterial roads and traffic count's locations. 


\subsection{Model Inputs}

The propsoed model requires the actual flows of the link $a$ as an input that must be placed in the objective function. Since this method is concerned with the freight O-D matrix as vehicle flow, these flows of links must be inserted to the model as the number of truck (2-axle and 3-axle and more). These online data are taken in 1165 points in Iran. Fig. 4 shows traffic count's locations. On the other hand, the model requires an initial freight O-D matrix. This matrix was also achieved with field studies in 2011.

\subsection{Zoning}

The first step for any transportation planning activities is zoning. As there is not any special rule in this area, different methods have been considered by many researchers (Martínez et al., 2007). In this paper, first, the production and freight absorption zones in which economic activities are done causing production and freight traffic absorption to/from other studied zones are specified. Each zone must have a center that is "center of gravity" of economic activities and all displacements from/to the zone can be measured there. Therefore, the considerations and criteria below have been used for defining zones. System of zones must be proportional with the country divisions, especially in related population and production statistics. Thus, from this perspective, the zones can be defined as: A series of smaller units that can be aggregated in different ways in different studies so that the results of these studies can be compatible and comparable with each other. Zones must be homogeneous as far as possible. This applies to urban zones because they are smaller zones based on the principle of homogeneity defined zones or travel purposes. This matter is easy in urban zones because these zones are smaller and are defined based on the homogeneity principle of zones or travel purposes. This is somewhat more difficult in intercity studies because zones are larger and each also includes a variety of different facilities or residents. Hence, homogeneity must be balanced based on the objectives of the study and 2 variables of the zone size and the units of the country divisions. To determine the border of zones, main roads should not be used and trip origins and destinations should not be on the border of zones, because this issue creates problem for traffic assignment in the modeling process. Regions must reflect the normal sphere of influence of its centrality as well as internal networks of the zone. This must be also visible in the appearance of zone in a way that shows the internal features of the zone as well. Zones should not necessarily be the same size, but their size should be proportional with the trip time units. Therefore, the more congested zones must have smaller dimensions. Chang et al. (2002) emphasized on the importance of this issue in estimation of the O-D matrix. Each zone is characterized by a balance point or center of gravity. In urban studies, centers of gravity are virtual points representing the average cost of trip to anywhere else in the region.

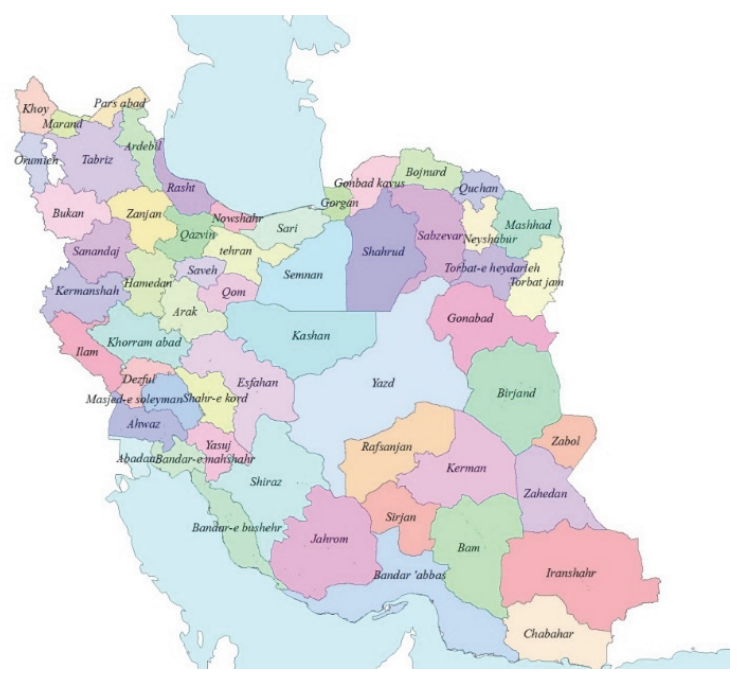

Fig. 5. Iran zoning 
These points are usually dependent to a particular site, but it is not necessarily so. These points are connected to the network by means of a link (virtual link) that reflects the average cost of connection to a node in the actual network (road). In intercity studies that larger zones are used, this process is vice versa. It means that first the central point which represents production and trip absorption centers are determined. Then, the zone border is defined and determined based on the influence sphere in central points of adjacent zones. Finally, after collecting the necessary the data and political divisions, Iran is divided into 56 zones while there are 31 provinces in Iran. On the other hand, in the studies conducted by Lim et al. (2014) it has been pointed out that a country like the United States of America could be divided into 114 zones for freight transport analyses. This indicates that by considering the volume of internal freight passing through both countries and their areas, 56 zones are appropriate for Iran with regard to available data restrictions. Finally, Iran zoning is shown as Fig. 5.

\subsection{Results}

To solve the model (1) in addition to process the input data, $\gamma_{1}$ and $\gamma_{2}$ values must be determined as well. The objective function above has considered deviation of the estimated values from the observed data for two types of data. $\gamma_{1}$ is the deviation factor in the O-D matrix of observed and estimated freight, while $\gamma_{2}$ is the deviation factor in traffic volume count of observed and estimated trucks; therefore, these factors should be determined to solve the model. For this purpose, several simulations from the model were conducted for different combinations of $\gamma_{1}$ and $\gamma_{2}$. This assumption was led to $\gamma_{1}=100-$ $\gamma_{2}$. This combination will cause the objective function is obtained by the minimum value.

As mentioned above and by considering the research conditions and existing data, the combination of $\gamma_{1}$ and $\gamma_{2}$ were selected with percentage of 50\%. Zargari and Hamedani (2006) also achieved these coefficients through field surveys. These values in the freight O-D matrix provide the best response. Finally, it was achieved by the assignment of final estimated matrix to the network of freight flows in network links. Fig. 6 displays the correspondence between the two-axle trucks passing from 100 links that have the highest passing volumes, based on observations and computational values obtained from the optimal freight O-D matrix assignment to the network. The fitted line has a correlation coefficient of 0.85 , which demonstrates the suitability of the results. Fig. 7 also illustrates one-to-one correspondence of the initial freight O-D matrix and the final matrix for 100 top pairs as shown in the graph. The correlation coefficient of the fitted line is 0.89 , which seems to be acceptable result of the freight matrix estimation. After using the propsoed model, its results should be examined. In this study, the volume of observed and estimated traffic must be analyzed, too. There are numerous parameters for this purpose such as Mean Absolute Percentage Error (MAPE), Root Mean Square Error (RMSE), and Mean Absolute Error (MAE) that can display the differences between observations and calculations. Moreover, there is also another parameter called GEH, which was defined by Geoffrey Havers for the first time. This index is defined based on ADT by (Horowitz et al., 2014), as follows:

$$
\mathrm{GEH}=\sqrt{\frac{0.2 M^{2}-0.4 M C+0.2 C^{2}}{M+C}}
$$

where:

$\mathrm{M}=$ Estimated Traffic Volume, ADT, and C = Observed Traffic Count (ADT).

One advantage of this parameter is that it can be applied in the issues related to the projections of traffic volume in transportation planning. For comparison of the observed and estimated daily traffic is the parameter defied as follows:

This parameter is calculated separately for each link and its results are interpreted as follows:

$$
\text { GEH }<5 \quad \text { Acceptable fit, probably OK }
$$


$5<\mathrm{GEH}<10 \quad$ Caution: possible model error or bad data

GEH $>10 \quad$ Warning: high probability of model error or bad data

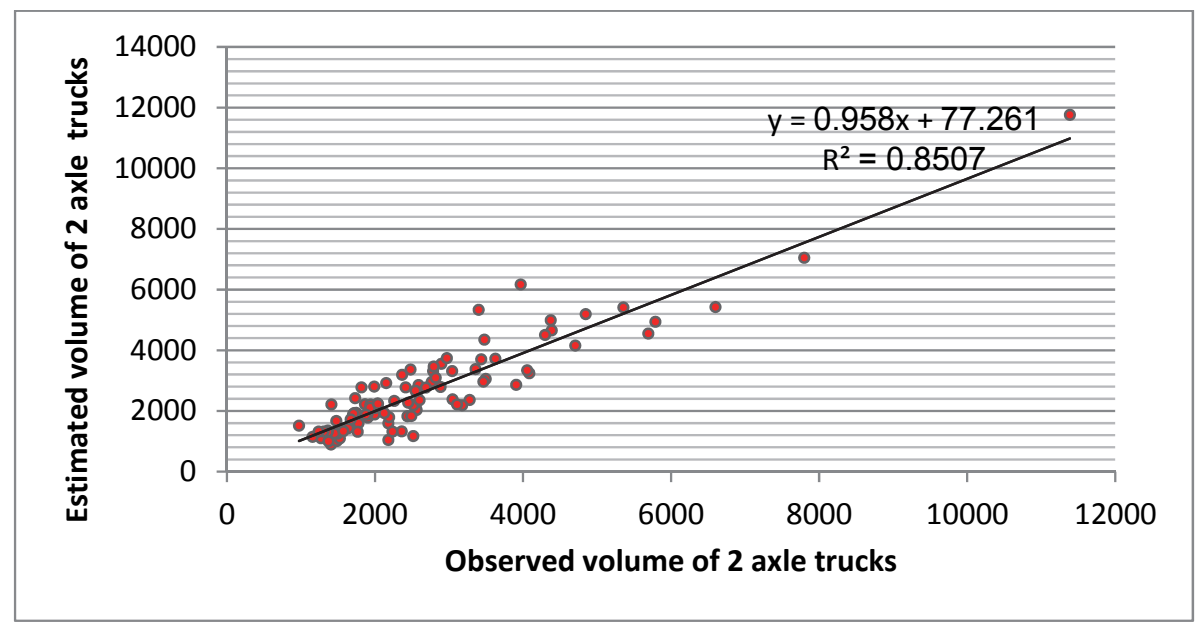

Fig. 6. Observed volume of 2-axle trucks against the estimated ones (veh/day)

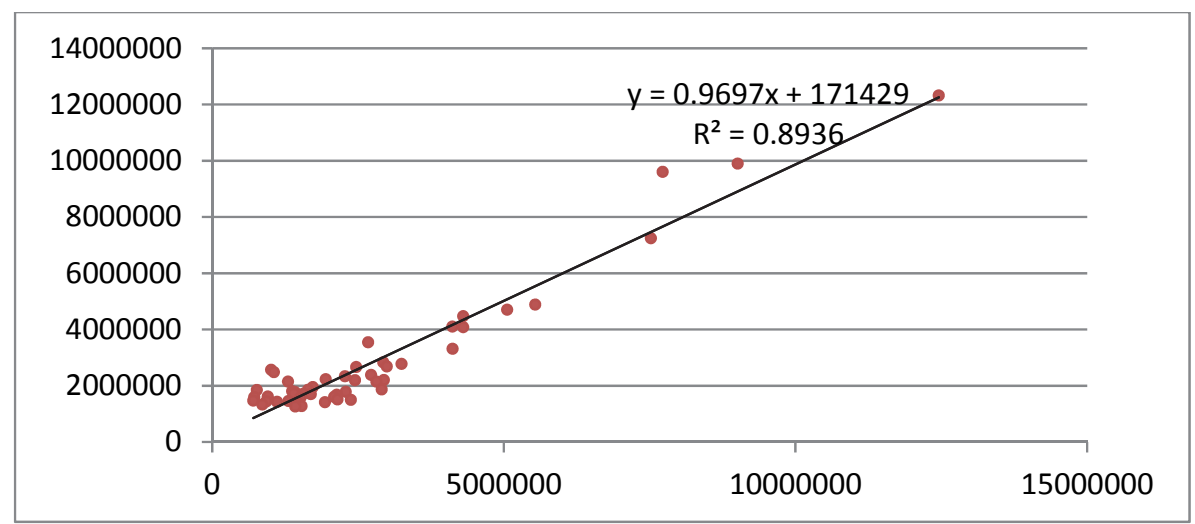

Fig. 7. Initial O-D matrix against the estimated ones for top 100 pairs (ton/year)

The results of this parameter for network links as well as trucks with two, three, or more axels are displayed in Table 1 and Table 2.

\section{Table 1}

Analysis of GEH function for average traffic of two-axle trucks per day

\begin{tabular}{lccc}
\hline GEH & Frequency & Cumulative & Cumulative $\%$ \\
\hline $0-0.5$ & 225 & 225 & $19 \%$ \\
$0.5-1.0$ & 169 & 394 & $34 \%$ \\
$1.0-1.5$ & 130 & 524 & $45 \%$ \\
$1.5-2.0$ & 144 & 668 & $57 \%$ \\
$2.0-2.5$ & 109 & 777 & $67 \%$ \\
$2.5-3.0$ & 70 & 847 & $73 \%$ \\
$3.0-4.0$ & 123 & 970 & $83 \%$ \\
$4.0-5.0$ & 75 & 1045 & $90 \%$ \\
$5.0-7.0$ & 72 & 1117 & $96 \%$ \\
$7.0-9.0$ & 29 & 1146 & $98 \%$ \\
$9.0-16.0$ & 19 & 1165 & $100 \%$ \\
\hline
\end{tabular}

Table 2

Analysis of GEH function for average traffic of three-axle trucks or more per day

\begin{tabular}{lccc}
\hline GEH & Frequency & Cumulative & Cumulative $\%$ \\
\hline $0-0.5$ & 153 & 153 & $13 \%$ \\
$0.5-1.0$ & 97 & 250 & $21 \%$ \\
$1.0-1.5$ & 112 & 362 & $31 \%$ \\
$1.5-2.0$ & 121 & 483 & $41 \%$ \\
$2.0-2.5$ & 139 & 622 & $53 \%$ \\
$2.5-3.0$ & 93 & 715 & $61 \%$ \\
$3.0-4.0$ & 151 & 866 & $74 \%$ \\
$4.0-5.0$ & 87 & 953 & $82 \%$ \\
$5.0-7.0$ & 80 & 1033 & $89 \%$ \\
$7.0-11.0$ & 132 & 1165 & $100 \%$ \\
\hline
\end{tabular}

The results are analyzed as follows: 
As specified in Tables 1 and 2, in the estimation of two-axel trucks in $87 \%$ of the links, GEH function is less than 5 . This index is $82 \%$ for three-axel trucks or more which indicates the efficacy of the results.

\section{Conclusion}

In this paper, a method has been provided for estimating the freight O-D matrix based on the traffic volume counts of commercial vehicles in the network links. This method is based on the minimization of least squares between the initial freight O-D matrix and the freight flow in links. It is clear that the freight exchange between zones in a country has a quite direct relationship with the freight commercial vehicle traffic including types of trucks (2-axle, 3-axle and more). Given that in 1165 points in Iran, there are traffic counters that quite online count the information based on vehicle types, these data and other information that are obtained from periodic surveys and somehow are related to the freight exchange between zones can be used. The freight O-D matrix can be estimated in intervals of less than a year as well. To solve this model, the meta-heuristic Simulated Annealing Algorithm has been employed. Although other meta-heuristic algorithms can be used in future studies to compare the results, the results obtained from solving the model with Simulated Annealing Algorithm indicate that to a large extent results are acceptable. This issue has been verified by comparing the correlation coefficient of the observed freight flow vector in network links and the computational freight flow obtained from the model solution as well as the correlation coefficient of the initial matrix obtained from the survey and the estimated freight O-D matrix obtained from the model solution.

\section{References}

Al-Battaineh, O., \& Kaysi, I. (2005). Commodity-based truck origin-destination matrix estimation using input-output data and genetic algorithms.Transportation Research Record: Journal of the Transportation Research Board, 1923, 37-45.

Horowitz, A., Creasey, T., Pendyala, R., \& Chen, M. (2014). Analytical Travel Forecasting Approaches for Project-level Planning and Design (No. Project 08-83).

Bell, M. G. (1991). The estimation of origin-destination matrices by constrained generalised least squares. Transportation Research Part B: Methodological, 25(1), 13-22.

Cascetta, E., \& Nguyen, S. (1988). A unified framework for estimating or updating origin/destination matrices from traffic counts. Transportation Research Part B: Methodological, 22(6), 437-455.

Cerny, V. (1985). Thermodynamical approach to the traveling salesman problem: An efficient simulation algorithm. Journal of optimization theory and applications, 45(1), 41-51.

Cohen, H. (1995). Future directions for freight modeling. In Proceedings of the urban goods and freight forecasting conference.

Chang, K. T., Khatib, Z., \& Ou, Y. (2002). Effects of zoning structure and network detail on traffic demand modeling. Environment and Planning B: Planning and design, 29(1), 37-52.

Crainic, T., Dufour, G., Florian, M., Larin, D., \& Leve, Z. (2001). Demand matrix adjustment for multimodal freight networks. Transportation Research Record: Journal of the Transportation Research Board, (1771), 140-147.

Flaskou, M., Dulebenets, M. A., Golias, M. M., Mishra, S., \& Rock, R. M. (2015). Analysis of Freight Corridors Using GPS Data on Trucks.Transportation Research Record: Journal of the Transportation Research Board, (2478), 113-122.

Jayaraman, V., \& Ross, A. (2003). A simulated annealing methodology to distribution network design and management. European Journal of Operational Research, 144(3), 629-645.

Jansuwan, S., Ryu, S., \& Chen, A. (2016). A two-stage approach for estimating a statewide truck trip table. Transportation Research Part A: Policy and Practice.

Khan, T., \& Anderson, M. (2016). Accurately Estimating Origin/Destination Matrices in Situations with Limited Traffic Counts: Case Study Huntsville, AL. International Journal of Traffic and Transportation Engineering, 5(3), 64-72.

Kirkpatrick, S., Gelatt, C. D., \& Vecchi, M. P. (1983). Optimization by simulated annealing. science, 220(4598), 671-680. 
Lim, R., Qian, Z. S., \& Zhang, H. M. (2014). Development of a Freight Demand Model with an Application to California. International Journal of Transportation Science and Technology, 3(1), 19-38.

List, G. F., \& Turnquist, M. A. (1994). Estimating truck travel patterns in urban areas. Transportation Research Record, (1430), 1-9.

List, G., Konieczny, L., Durnford, C., \& Papayanoulis, V. (2002). Best-practice truck-flow estimation model for the New York City region.Transportation Research Record: Journal of the Transportation Research Board, (1790), 97-103.

Ma, X., McCormack, E., \& Wang, Y. (2011). Processing commercial global positioning system data to develop a web-based truck performance measures program. Transportation Research Record: Journal of the Transportation Research Board, (2246), 92-100.

Maher, M. J. (1983). Inferences on trip matrices from observations on link volumes: a Bayesian statistical approach. Transportation Research Part B: Methodological, 17(6), 435-447.

Martínez, L., Viegas, J., \& Silva, E. (2007). Zoning decisions in transport planning and their impact on the precision of results. Transportation Research Record: Journal of the Transportation Research Board, (1994), 58-65.

Metropolis, N., Rosenbluth, A. W., Rosenbluth, M. N., Teller, A. H., \& Teller, E. (1953). Equation of state calculations by fast computing machines. The journal of chemical physics, 21(6), 1087-1092.

Pinjari, A., Short, J., Pierce, D., Park, L., Murray, D., Mysore, V., ... \& Irmania, A. (2013). Truck GPS Data for Freight Performance Measurement, Modeling and Planning.

Poorjafari, V., Yue, W. L., \& Holyoak, N. (2014). Application of simulated annealing in transit schedule synchronization. International Journal of Modeling and Optimization, 4(6), 476.

Réos, A., Nozick, L., \& Turnquist, M. (2002). Value of different categories of information in estimating freight origin-destination tables. Transportation Research Record: Journal of the Transportation Research Board, (1783), 42-48.

Shabani, K., \& Figliozzi, M. (2012, January). A statistical study of commodity freight value/tonnage trends in the United States. InTransportation Research Board 91st Annual Meeting Compendium of Papers, Washington DC, United States.

Spiess, H. (1987). A maximum likelihood model for estimating origin-destination matrices. Transportation Research Part B: Methodological, 21(5), 395-412.

Tamin, O. Z., \& Willumsen, L. G. (1989). Transport demand model estimation from traffic counts. Transportation, 16(1), 3-26.

Van Zuylen, H. J., \& Willumsen, L. G. (1980). The most likely trip matrix estimated from traffic counts. Transportation Research Part B: Methodological, 14(3), 281-293.

Wang, Y., Ma, X., Liu, Y., Gong, K., Henricakson, K. C., Xu, M., \& Wang, Y. (2016). A Two-Stage Algorithm for Origin-Destination Matrices Estimation Considering Dynamic Dispersion Parameter for Route Choice. PloS one,11(1), e0146850.

Woch, M., \& Łebkowski, P. (2009). Sequential simulated annealing for the vehicle routing problem with time windows. Decision Making in Manufacturing and Services, 3(1-2), 87-100.

Zargari, S. A., \& Hamedani, S. Y. (2006). Estimation of freight OD matrix using waybill data and traffic counts in Iran roads. Iranian Journal of Science \& Technology, Transaction B, Engineering, 30(B1).

Zhang, Y., Bowden, R. O., \& Allen, A. J. (2004). Intermodal freight transportation planning using commodity flow data (No. Final Research Report). United States Department of Transportation, Research and Special Programs Administration.

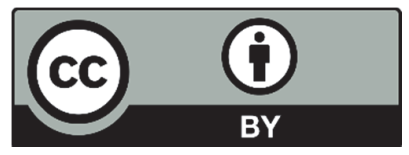

(C) 2018 by the authors; licensee Growing Science, Canada. This is an open access article distributed under the terms and conditions of the Creative Commons Attribution (CC-BY) license (http://creativecommons.org/licenses/by/4.0/). 